\title{
El desarrollo de la actividad física con adultos obesos del Municipio Nueva Paz
}

The development of physical activity with obese adults of the Nueva Paz Municipality

Efraín Velasteguí López. ${ }^{1}$ Daylen de la C Domínguez Sánchez. ${ }^{2}$ Laura Diaz Torres. ${ }^{3}$ Glenda González Calzadilla. ${ }^{4}$ Meibis Morgados Martínez. ${ }^{5}$ \& Nilian González del Pino. ${ }^{6}$

\section{https://doi.org/10.33262/anatomiadigital.v3i1.1120}

\section{Abstract.}

The poor training of physical culture teachers does not allow physical activity to develop adequately with obese adults. This problem determined the scientific problem of this research. How to favor the preparation of teachers of physical culture for the

\section{Resumen.}

La deficiente capacitación de los profesores de cultura física no permite que se pueda desarrollar adecuadamente la actividad física con personas obesas de edad adulta. Esta problemática determinó el problema científico de esta investigación ¿Cómo

1 Ciencia digital, Ambato, Ecuador, luisefrainvelastegui@cienciadigital.org https://orcid.org/0000-0002-7353-0209

2 Ciencias Médicas Mayabeque, Mayabeque, Cuba, daydominguez@infomed.sld.cu https://orcid.org/0000-0002-7353-0210

${ }^{3}$ Ciencias Médicas Mayabeque, Mayabeque, Cuba, ldiaz@infomed.sld.cu iD https://orcid.org/0000-00027353-0211

${ }^{4}$ Ciencias Médicas Mayabeque, Mayabeque, Cuba, glengonzalez@infomed.sld.cu ID https://orcid.org/00000002-7353-0212

${ }^{5}$ Ciencias Médicas Mayabeque, Mayabeque, Cuba, meimorgados@infomed.sld.cu iD https://orcid.org/00000002-7353-0213

${ }^{6}$ Ciencias Médicas Mayabeque, Mayabeque, Cuba, ngonzalez@infomed.sld.cu iD https://orcid.org/00000002-7353-0214 
development of physical activity with obese adults of the Nueva Paz Municipality? The objective of this research that responds to the scientific problem was: Develop workshops for the training of teachers of physical culture for the development of physical activity with obese adults of the Nueva Paz Municipality. The workshops were selected, after studying various organizational forms of teacher training, taking into account that they, from their own dynamics, stimulate the role of independent study individually, independently self-evaluate during the course of their teaching process. A total of seven workshops were planned. The facilitator is the researcher, the participants are all the physical culture teachers to whom the proposed workshops will be applied. To evaluate the efficiency of the workshops, a type of evaluation is chosen that will focus on the process, progress and results.

Keywords: deficiency, training. favorecer la preparación de profesores de cultura física para el desarrollo de la actividad física con adultos obesos del Municipio Nueva paz? El objetivo de esta investigación que da respuesta al problema científico fue: Elaborar talleres para la capacitación de los profesores de cultura física para el desarrollo de la actividad física con adultos obesos del Municipio Nueva paz.

Se seleccionaron los talleres, después del estudio de variadas formas organizativas de capacitación para docentes, atendiendo a que ellos, desde su propia dinámica, estimulan el protagonismo el estudio independiente de forma individual, se autoevalúan independientemente en el transcurso del proceso de su superación docente. Se planificaron un total de siete talleres. El facilitador es el investigador, los participantes son todos los profesores de cultura física a los que se les va a aplicar los talleres propuestos. Para evaluar la eficiencia de los talleres se opta por un tipo de evaluación que se centrará en el proceso, avances y resultados.

Palabras claves: deficiencia, capacitación.

\section{Introducción.}

La obesidad es una condición patológica, muy común en el ser humano y presente desde la más remota antigüedad, que persiste y se incrementa durante siglos por factores genéticos y ambientales, hasta convertirse actualmente en una pandemia con consecuencias nefastas para la salud.

Al realizar un breve repaso de la evolución de la actividad física cotidiana del ser humano, vemos que esto está vinculado a la obesidad desde el hombre primitivo hasta la época actual, resulta que a partir del descubrimiento de la rueda se inició un cambio de conducta en los desplazamientos y en la misma obtención de alimento, posteriormente con la revolución industrial aparece la máquina de vapor que contribuyó a la mecanización de la agricultura y 
al desarrollo de la industria automovilística transformándose el hombre en una persona sedentaria en extremo.

Por otra parte también se observa una radical modificación en la conducta nutricional desde los primeros seres humanos hasta nuestros días.

En la alimentación de los primeros seres humanos los productos de origen animal no superaban el $20 \%$ de la ración diaria, el resto era vegetal. El salado el ahumado y el secado al sol fueron los primitivos métodos de conservar las carnes, verduras, y frutas, la dieta primitiva se completaba con leche cuajada (yogurt).

$\mathrm{Al}$ extenderse la cultura medieval se incrementa notablemente la agricultura y la ganadería en pequeñas comunidades, luego de tantos siglos de comer así en sociedad de pastores y agricultores en los últimos doscientos años contamos con muchos más alimentos.

En la dieta moderna de la sociedad desarrollada, las grasas animales y el azúcar, llegan a ocupar la mitad de la ración.

La vida ciudadana presente trajo aun más cambios, todos comen lo que publicitan los medios masivos de la comunicación. La comida es de alta densidad energética basada en animales, azucares y harinas refinadas, quedando poco lugar para los granos y las verduras. El consumo de sal es diez veces mayor que el de nuestros antepasados y la comida llega con aditivos químicos (solventes orgánicos y plásticos).

En la actualidad se estima el número de personas obesas en el mundo es más de 300 millones, con una amplia distribución mundial y una prevalencia mayor en países desarrollados o en vías de desarrollo.

El sobrepeso y la obesidad, es una epidemia que afecta al individuo de todas las edades, sexos, razas y latitudes, sin respetar el nivel socioeconómico. Es la consecuencia de la existencia de un balance energético positivo que se prolonga durante un tiempo. Tratándose de la obesidad es una obligación de todos unirse para enfrentarla y dominarla.

El exceso de consumo de alimentos ricos en grasas o densos en calorías unidos al habito de poca actividad física, asociados a factores hereditarios, neurológicos, culturales y sociales entre otros pueden predisponer y condicionar en la persona un aumento de peso corporal a expensa de grasa de depósito.

Este aumento de peso producido por los elementos antes expuestos, propician el síndrome endocrino de la obesidad y constituye un factor de riesgo de gran significación e importancia en el desarrollo de afecciones que presentan elevadas tasas de mortalidad, como son: la hipertensión arterial, la diabetes mellitas, la aterosclerosis, entre otras. 
En Cuba a pesar de ser un país del tercer mundo se observan fenómenos del primer mundo entre ellos la obesidad y sus enfermedades asociadas por lo cual se hace necesario conocerlas y combatirlas.

Elevar la calidad de vida de la población y evitar tan penosas enfermedades son objetivos del INDER y el MINSAP, creándose el programa de la obesidad, por el cual se pretende incorporar a la práctica de ejercicio físico de forma sistemática a la población sana y a los que padezcan enfermedades como la obesidad, la hipertensión y otras propias del resultado de una vida sedentaria.

En visitas y el diagnostico realizados por el INDER y el MINSAP a los consultorios médicos de la familia se detecta una alta cantidad de población con tendencia a la obesidad además de una insuficiente labor por parte de los profesores para desarrollar la actividad física con adultos obesos del municipio Nueva Paz.

Todas estas deficiencias se manifiestan en:

- Una inadecuada preparación de los profesores de cultura física para el desarrollo de la actividad física con adultos obesos.

- Existencia de una pobre asistencia de estas personas obesas a las actividades planificadas y organizadas.

\section{Desarrollo}

La investigación tiene su sustento teórico y metodológico en la confección de talleres de capacitación que favorecen el nivel de conocimientos y la actualización sobre el tema, en el marco de los restantes procesos sociales. Donde se establece la metodología que permite aportar el resultado científico. Es una investigación de tipo descriptiva exploratoria, de corte cualitativo y en su concreción se tuvo en cuenta la utilización de los métodos teóricos, dentro de ellos: el histórico lógico, análisis y síntesis, y la inducción y deducción. Como métodos empíricos: se utilizó la observación participante, el análisis documental.

\section{Consideraciones acerca de la capacitación.}

"La capacitación significa la preparación de la persona en el cargo, en tanto que el propósito de la educación es preparar a la persona para el ambiente dentro o fuera de su trabajo." La "capacitación" es la educación profesional que busca adaptar al hombre para determinada empresa.

Definición de capacitación. "Se entiende por capacitación el conjunto de procesos organizados, relativos tanto a la educación formal como a la informal de acuerdo con lo establecido por la ley general de educación, dirigidos a prolongar y a complementar la 
educación inicial mediante la generación de conocimientos, el desarrollo de habilidades y el cambio de actitudes, con el fin de incrementar la capacidad individual y colectiva para contribuir al cumplimiento de la misión institucional, a la mejor prestación de servicios a la comunidad, al eficaz desempeño del cargo y al desarrollo personal integral “. Según Capacitación.htm.

En Cuba el Reglamento para la planificación, ejecución y control del trabajo de la capacitación y desarrollo de los recursos humanos, en las entidades laborales, en su capítulo I, artículo \# 2 se define capacitación como: "Conjunto de acciones de preparación que desarrollan las entidades laborares dirigidas a mejorar las competencias, calificaciones y recalificaciones para cumplir con calidad las funciones del puesto de trabajo y alcanzar los máximos resultados productivos o de servicios. Este conjunto de acciones permite crear, mantener y elevar los conocimientos, habilidades y actitudes de los trabajadores para asegurar su desempeño exitoso".

En esta investigación se asume la concepción de ubicar la capacitación dentro del proceso educativo de la Cultura Física y para ello se parte del análisis de la coincidencia que existe entre diferentes autores cubanos, en cuanto a la dimensión del término educación, por lo que frecuentemente se encuentra que para referirse al mismo, parten de la definición de educar dada por José Martí: "Educar es depositar en cada hombre toda la obra humana que le ha antecedido: es hacer a cada hombre resumen del mundo viviente, hasta el día en que vive: es ponerlo al nivel de su tiempo, para que flote sobre él, y no dejarlo por debajo de su tiempo, con lo que no podrá salir a flote: es preparar al hombre para la vida”. (Martí, 1961).

Los principales objetivos de la capacitación son:

1. Preparar al personal para la ejecución de las diversas tareas particulares de la organización.

2. Proporcionar oportunidades para el continuo desarrollo personal, no sólo en sus cargos actuales sino también para otras funciones para las cuales la persona puede ser considerada.

3. Cambiar la actitud de las personas, con varias finalidades, entre las cuales están crear un clima más satisfactorio entre los empleados, aumentar su motivación y hacerlos más receptivos a las técnicas de supervisión y gerencia.

\section{La capacitación de los profesores de cultura física.}

En los espacios académicos hoy se produce una animada discusión sobre términos como capacitación, preparación y superación. En algunos casos se tienden a identificar como sinónimos entre otros, como términos con gran significación semántica diferente. Algunos autores se sustentan en reglamentos y en otros documentos normativos, sin realizar una fundamentación teórica en los términos aludidos. Es cierto que, de acuerdo con la posición 
asumida por los autores consultados, dichos términos pueden considerarse unos dentro de otros.

El profesor de cultura física requiere de una constante superación, que lo mantenga actualizado en las cuestiones fundamentales de su quehacer. Esto se posibilita cuando existe una capacitación bien concebida y enfocada hacia aquellos aspectos de la labor físico cultural que necesitan de una mayor atención.

En el caso de este aspecto en el municipio, se realizan actividades de carácter metodológico y de superación y se han impartido diversos seminarios no siendo suficiente para el caso de los programas priorizados referentes a las enfermedades crónicas no transmisibles en el caso que nos ocupa la obesidad.

\section{Referentes teóricos acerca de la Obesidad.}

En esta investigación no asumimos un criterio exacto, acerca del tema entendemos que con varios aspectos tenemos una definición propiamente amplia.

La obesidad solo es debida a trastornos del sistema endocrino en contadas ocasiones. En la mayor parte de los casos, la obesidad es la consecuencia de un aporte de energía a través de los alimentos que supera al consumo de energía a través de la actividad, este exceso de calorías se almacena en el cuerpo en forma de grasa. La obesidad puede deberse también a la falta de actividad, como sucede en personas sedentarias o postradas en la cama.

\section{Los talleres como formas organizativas de la capacitación de los profesores de cultura física.}

En la actualidad se denomina taller a las más diversas formas de estructurar las actividades del proceso de enseñanza aprendizaje, no solo en el desarrollo de la formación de diversos especialistas, sino también en su capacitación. En todos los autores consultados es común el criterio de que el objetivo central del taller es la práctica, la discusión, el debate y el pensamiento crítico con un enfoque vivencial.

En este trabajo se asume taller como la forma de organizar actividades de capacitación en un grupo de profesores de cultura física en un tema determinado y a partir de un objetivo, en función de distribuir la dinámica colectiva de sus integrantes para resolver un problema en la práctica de la profesión (en este caso el desarrollo de la actividad física con adultos obesos).

Ventajas del taller como forma organizativa:

- Los problemas se solucionan mejor cuando se está en grupo, lo que despierta una mayor divergencia del pensamiento, o sea, un mismo problema necesita de variadas respuestas. 
- Es una estructura organizada centrada en la reflexión grupal sobre los problemas. La reflexión no da paso a la improvisación, sino que tiene que estar combinada la teoría con la práctica.

- Las soluciones en grupo incitan a los procesos creativos de la realidad, se combina la creatividad individual con la colectiva.

- Integra y combina la teoría con la práctica, la producción y asimilación de conocimientos, habilidades, hábitos, valores, y su reflejo en la ética profesional, entre lo temático y lo dinámico en las relaciones interpersonales, entre la investigación y la docencia.

- Se parte de una problemática común detectada en la práctica pedagógica.

- Exige alto nivel de participación y una redimensión de sus actuales saberes para buscar soluciones de calidad que transforme la realidad.

- Expresa la relación dialéctica entre la organización y el sujeto, dicha relación es de carácter interna, o sea los talleres pueden variar el contenido de sus componentes de acuerdo con las necesidades y particularidades de cada institución.

- Rechaza la reproducción mecánica y el no protagonismo a los actores de los procesos.

En el taller los resultados dependen no solo de lo que se pueda transmitir a los profesores de cultura física sino de la actividad que sean capaces estos de desempeñar para el logro de los objetivos propuestos. El que facilita la conducción de la actividad asume la función de orientador y guía, y los talleristas aprenden haciendo reflexiones colectivas y proponiendo soluciones valiosas, que resultan de gran relevancia para el trabajo colectivo, donde cada cual revela su experiencia en la materia que se trata. Además, el taller es una vía eficaz para desarrollar la capacitación de los profesores de cultura física, lo que constituye la médula para la elaboración de la propuesta que se realiza.

\section{Propuesta.}

\section{Fundamentación de la propuesta.}

La propuesta talleres de capacitación a profesores de cultura física para el desarrollo de la actividad física con adultos obesos municipio Nueva Paz se fundamenta en la necesidad de solucionar un problema que se ha presentado por una situación que no es ajena a los involucrados en esta investigación, es contradictoria la necesidad de desarrollar la actividad física en adultos obesos y la falta de preparación que tienen los profesores de cultura física para el desarrollo de esta.

Con el fin de resolver tal situación tratamos de mejorar el servicio prestado por los profesores de cultura física brindando la mayor satisfacción y bienestar a las personas, este es uno de 
nuestros compromisos y responsabilidades de manera que no resulta insólito que uno de los objetivos principales sea:

Capacitar a los profesores para perfeccionar la organización y el desarrollo de su servicio en función de mejorar del bienestar físico y mental de las personas que presentan obesidad en edad adulta.

\section{Dosificación de los talleres}

Se concibieron cuatro temas, cada uno con sus temáticas y un total de siete talleres, como queda reflejado en la tabla No1

Tabla 1. Temas, con sus temáticas de los talleres

\begin{tabular}{|c|l|l|c|}
\hline No & \multicolumn{1}{|c|}{ TEMAS } & \multicolumn{1}{|c|}{ TEMÁTICAS } & HORAS \\
\hline 1 & Encuadre. & $\begin{array}{l}\text { Presentación de los participantes. Socialización de la } \\
\text { propuesta de talleres para la capacitación. }\end{array}$ & 2 \\
\hline 2 & La obesidad. & $\begin{array}{l}\text { Antecedentes históricos de la obesidad. Etiología. } \\
\text { Clasificaciones. }\end{array}$ & 2 \\
\hline 3 & La obesidad. & $\begin{array}{l}\text { Diferentes clasificaciones de la obesidad. } \\
\text { Peculiaridades. }\end{array}$ & 2 \\
\hline 4 & $\begin{array}{l}\text { La actividad física y y } \\
\text { la obesidad. }\end{array}$ & $\begin{array}{l}\text { Acerca de la actividad física. Influencia de la } \\
\text { actividad física en el adulto obeso. Programa que } \\
\text { trata la obesidad. }\end{array}$ & 2 \\
\hline 5 & $\begin{array}{l}\text { La actividad física y y } \\
\text { la obesidad. }\end{array}$ & $\begin{array}{l}\text { Peculiaridades psicológicas del obeso. El desarrollo } \\
\text { de la motivación por la actividad física en el adulto } \\
\text { obeso. }\end{array}$ & 2 \\
\hline 6 & $\begin{array}{l}\text { La actividad física y } \\
\text { la obesidad. }\end{array}$ & $\begin{array}{l}\text { Ejercicios físicos recomendados para el adulto } \\
\text { obeso. Requisitos. }\end{array}$ & 2 \\
\hline 7 & Cierre de los talleres. & \begin{tabular}{l} 
Evaluación de los usuarios acerca de los talleres. \\
\hline
\end{tabular} & 2.30 \\
\hline
\end{tabular}

Fuente: elaboración propia

Los objetivos generales de los talleres planteados son:

- Capacitar a los profesores de cultura física acerca del desarrollo de la actividad física con adultos obesos, a partir de concepciones de carácter teórico y práctico.

- Analizar las actividades físicas que favorecen el mejoramiento de la salud de los adultos obesos. 
Las vías que se emplean para el desarrollo de los talleres pedagógicos son: seminarios, intercambio de experiencia, trabajo de mesa, debates y actividad demostrativa.

Para la cantidad de horas de los talleres se debe tener en cuenta la preparación previa y la orientación que los profesores de cultura física deben alcanzar, para luego entrar en la discusión de cada una de las temáticas correspondientes a cada tema.

En todos los talleres el facilitador debe ser un profesional, de ser posible el propio investigador. Los participantes son los profesores de cultura física y en caso de creerlo necesario invitar a médicos de la familia, para las temáticas que tienen un fuerte componente teórico de la especialidad médica.

Para la evaluación se deben culminar todos los talleres y tener en cuenta:

- Valoración del comportamiento de los profesores de cultura física las temáticas abordadas.

- El cumplimiento de cada uno de los objetivos propuestos.

La evaluación se debe efectuar con la participación de los talleristas, incluyendo en el último al médico de la familia, al ejecutor del programa.

A continuación, se plantean los talleres propuestos:

\section{Taller 1: Encuadre}

Temática: Presentación de los participantes. Socialización de la propuesta de talleres para la capacitación.

Objetivo: Analizar la propuesta de talleres de capacitación con las técnicas de cultura física acerca del desarrollo de las actividades físicas con adultos obesos.

Via: Seminario

Tiempo: 2 horas

Desarrollo: La actividad comenzara con la presentación de los talleristas y el facilitador, con el fin de que cada uno exprese sus expectativas alrededor de los talleres que se desarrollan.

El grupo se dividirá en parejas y seguidamente se le entregara un material docente que contiene una síntesis de los presupuestos teóricos en los que se sustentan los talleres propuestos y el desarrollo de 2 de estos talleres. Se dará un tiempo de 40 minutos para escribir las consideraciones y pasar al debate 
En el debate se deben cuidar las reglas del trabajo en grupo y se les pedirá que viertan sus valoraciones sobre las propuestas que se les presento, revelando potencialidades y dificultades, así como las recomendaciones que proponen a los talleres.

Luego se procederá a evaluar cada participante, a partir de la toma de sus criterios. En este taller y en los siguientes se tendrán en cuenta las indicaciones planteadas para esta evaluación

Se orientará la temática de próximo taller.

Evaluación: Toma de criterios de los participantes.

\section{Taller 2: Obesidad.}

Temática: Antecedentes históricos de la obesidad. Etiología. Clasificaciones

Objetivo: Discutir acerca de los antecedentes históricos de la obesidad su etiología y clarificación

Via: Intercambio de experiencias

Tiempo: 2 horas

Desarrollo:

Revisión de la actividad orientada

Reflexionar acerca de la obesidad

¿Desde qué etapa del desarrollo histórico de la humanidad aparecen registrados los síntomas de la obesidad?

¿Cuáles son las particularidades de la obesidad?

¿Cómo se clasifica?

¿Qué vivencias tienen acerca de los aspectos tratados?

Evaluación: Toma de criterios de los participantes.

\section{Taller 3: Obesidad.}

Temática: Diferentes clasificaciones de la obesidad. Peculiaridades.

Objetivos: Analizar la clasificación de la obesidad, así como sus peculiaridades. 
Vía: Trabajo de mesa.

Tiempo: 2 horas

Desarrollo:

Comprobación del cumplimiento de la orientación dada en el taller anterior. Verificar que estén preparados convenientemente

Se puede utilizar la variante de conformar tríos para estudiar la bibliografía indicada.

Estudiar:

- Criterios de clasificación,

- Peculiaridades de cada una

- Causas

Posteriormente se debatirán los resúmenes, siguiendo las reglas de trabajo en grupo.

Se realizará la Orientación de las temáticas a trabajar en el próximo taller

"Acerca de la actividad física. Influencia de la actividad física en el adulto obeso. Programa que trata la obesidad."

Evaluación: Toma de criterios de los participantes.

\section{Taller 4: Actividad física y obesidad.}

Temática: Acerca de la actividad física. Influencia de la actividad física en el adulto obesidad. Programa que trata la obesidad.

Objetivos: Analizar las características de la actividad física y su influencia en le adulto obeso. Programa obesidad

Via: Trabajo de mesa

Tiempo: 2 horas

Desarrollo:

Al inicio de la actividad se realizará verificado si los talleristas pudieron prepararse para el taller 
Se procederá de forma similar al taller anterior, mediante las reglas de trabajo en grupo. Se deben formar los tríos de la misma manera que en el taller anterior para luego analizar textos.

Se debatirán resúmenes.

Orientación de la temática o trabajar en el próximo taller.

Peculiaridades psicológicas del obeso. Desarrollo de la motivación por la actividad física. Consultar varios textos.

Pueden escoger libremente la bibliografía a utilizar, se recomienda consultar páginas en la Internet y en las páginas de infomed.

Evaluación: Toma de criterios de los participantes.

\section{Taller 5: Obesidad.}

Temática: Peculiaridades psicológicas del obeso. El desarrollo de la motivación por la actividad física en el adulto obeso.

Objetivo: Valorar el comportamiento psicológico del obeso y las formas de promover su motivación hacia la actividad física.

Vía: Debate.

Tiempo: 2 horas

\section{Desarrollo:}

Comienza el taller con en control de la auto preparación de las talleristas. Preguntas:

¿Cuáles son las peculiaridades de la obesidad?

¿Cómo se comportan los obesos?

¿De qué manera se lograría controlar la obesidad?

¿Cómo puede ayudar la actividad física a un obeso?

¿Qué actitud tomar en cuanto a los tratamientos?

¿Por qué es importante que el técnico de Cultura Física conozca a cada uno de sus participantes?

¿Qué experiencias tener en cuanto a la práctica de la actividad física en sus participantes? 
¿De qué manera promueven la práctica de ejercicios físicos los profesores de Cultura Física?

¿Cómo lograr motivar a los adultos obesos?

El debate deberá respetar las reglas de trabajo en grupo, y promover a los talleristas a narrar sus vivencias.

Orientación de las temáticas del próximo taller "ejercicios recomendados para adultos obesos. Requisitos y aspectos a tener en cuenta". Mediante auto preparación.

Escogerán la bibliografía de la misma manera que en el taller anterior consultarán páginas o libros que estén actualizados en el tema.

Evaluación: Toma de criterios de los participantes.

\section{Taller 6: Ejercicios físicos para el obeso adulto.}

Temática: Ejercicios físicos recomendados para el adulto obeso. Requisitos.

Objetivo: Analizar las actividades físicas más recomendables para el adulto obeso y requisitos para su desarrollo.

Vía: Intercambio de experiencias

Tiempo: 2 horas

Desarrollo:

Se comenzará controlando la auto preparación de los talleres

Esta temática es muy importante, el joven técnico sin experiencias acerca de este programa especializado no conoce ejercicios que favorecen.

Seguidamente se presentará una pancarta con los siguientes aspectos sobre las cuales se realizará el intercambio de experiencias.

¿Qué actividades físicas programadas es más recomendable para el adulto obeso?

¿Cuáles son los requisitos para que los participantes intercambien experiencias?

Las respuestas dadas serán motivos de reflexión grupal y pueden ser profundizados por el investigador

Después de escuchar sus respuestas e intercambio se realiza la orientación de la temática a trabajar en el próximo taller. 
Evaluación: toma de criterios de los participantes.

\section{Taller 7:}

Temática: Evaluación de los usuarios acerca de los talleres.

Objetivo: Analizar la evaluación realizada por los profesores de cultura física a los talleres realizados.

Vía: Debate

Tiempo: 2:30 horas

Desarrollo: Los talleres se reunirán por pareja o trios y resumirán los criterios sobre los talleres en 3 columnas

- Positivo

- Negativo

- Interesantes

Una vez realizado el debate, se llevarán a una pizarra todos los criterios emitidos y se realizara un debate general.

Posteriormente se aplicarán las encuestas de usuarios, las que unidas a los criterios positivos, negativos e interesantes de los talleristas, se podrá realizar la evaluación general de los talleres. Estas serán tabuladas por los propios participantes.

Finalmente se evaluará la participación de cada tallerista.

Evaluación: Toma de criterios de los participantes.

\section{Conclusiones.}

Luego del estudio realizado. Se puede concluir que:

- Para la realización exitosa de las actividades físicas por parte del adulto obeso resulta necesario establecer talleres de capacitación con los profesores de Cultura física, elevando así la calidad de las actividades planificadas.

- Los resultados del diagnóstico aplicado en los consultorios atendidos por profesores en relación al comportamiento de la obesidad en la población neopacina denota la baja incorporación del adulto obeso a la práctica de las actividades físicas. 
- El estado actual de la capacitación de los profesores indica las necesidades de conocimiento para realizar actividades físicas programadas con adultos obesos.

\section{Referencias bibliográficas.}

Añorga Morales, J. 1995. La educación Avanzada: Una teoría para el mejoramiento profesional y humano. ISP. Enrique José Varona. La Habana. Cuba.

Añorga, Julia y col. (2008) Glosario de términos de la Educación Avanzada. La Habana: Formato digital.

Aroche Carvajal, A (2001) y Fleitas Rodríguez, L. M. (2009)

Aroche Carvajal, A., 2001.32

Arrula Adriana Carribero, Cedido por UCh RR.HH. portal de estudiantes de RR.HH Formación y capacitación del talento humano 06-2002

biblioteca.idict.villaclara.cu/UserFiles/File/.../rv0429.pdf

cubachile.com/index.php?...fisica...fisica...actividad-fisica.

Diccionario de la Lengua Española Océano Practico, 2000.

es.wikipedia.org/wiki/Obesidad

Fleitas Rodríguez L. M. 2009. Talleres pedagógicos sobre la actividad conjunta para docentes del tercer ciclo del círculo infantil. Tesis de Maestría (Maestría en Ciencias de la Educación). La Habana. Universidad de Ciencias Pedagógicas "Rubén Martínez Villena".

http://aceproject.org/main/espanol/et/etd05b01.htm

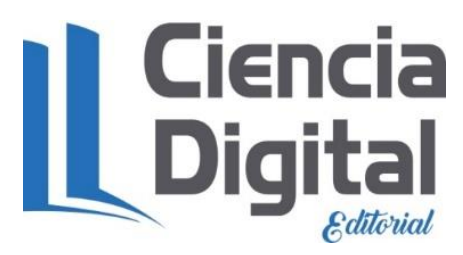




\section{PARA CITAR EL ARTÍCULO INDEXADO.}

Domínguez Sánchez, D. de la C., Diaz Torres, L., González Calzadilla, G., Morgados Martínez, M., \& González del Pino, N. (2020). El desarrollo de la actividad física con adultos obesos del Municipio Nueva Paz. Anatomía Digital, 3(1), 30-45. https://doi.org/10.33262/anatomiadigital.v3i1.1120

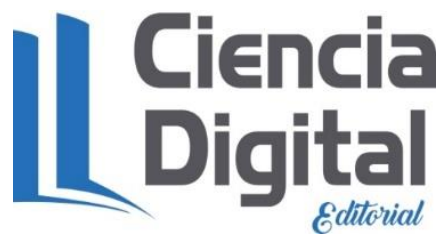

El artículo que se publica es de exclusiva responsabilidad de los autores y no necesariamente reflejan el pensamiento de la Revista Anatomía Digital.

El artículo queda en propiedad de la revista y, por tanto, su publicación parcial y/o total en otro medio tiene que ser autorizado por el director de la Revista Anatomía Digital.
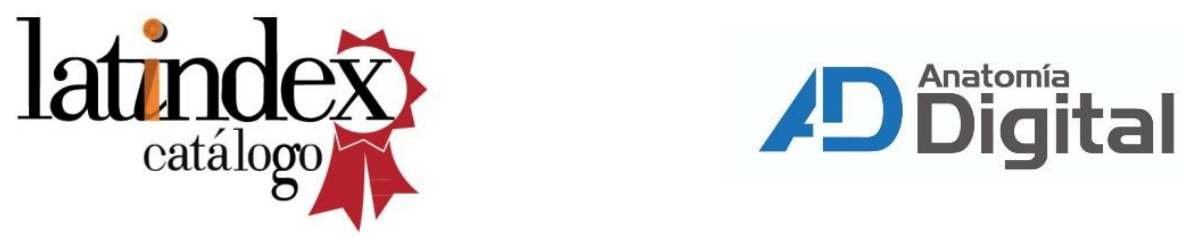